\title{
GALE NOTOEDRIC: UNE BRÈVE RÉVISION
}

\section{Cláudia Silva dos SANTOS ${ }^{1}$; Ytalo George NUNES ${ }^{2}$; Thiago Ribeiro BARBOSA ${ }^{3}$; Gil Dutra FURTADO $^{4}$; Leonardo Alves de FARIAS ${ }^{5}$; Magnolia Ouriques de OLIVEIRA ${ }^{6}$}

\author{
${ }^{1}$ Acadêmica de Medicina Veterinária/Centro Universitário Maurício de Nassau, João Pessoa (UNINASSAU/PB), \\ Brasil. E-mail: santos.claudia39@yahoo.com.br \\ ${ }^{2}$ Acadêmico de Medicina Veterinária/UNINASSAU/PB. E-mail: ytalo.geo@gmail.com \\ ${ }^{3}$ Acadêmica de Medicina Veterinária/UNINASSAU/PB. E-mail: thiago.r.barbosa@hotmail.com \\ ${ }^{4}$ Graduando em Medicina Veterinária/UNINASSAU/PB; Engenheiro Agrônomo/Universidade Federal da Paraíba \\ (UFPB), Brasil; Doutor em Psicobiologia/ Universidade Federal do Rio Grande do Norte (UFRN), Brasil; Sócio \\ da Cooperativa de Agronegócio (COOPAGRO), Brasil. E-mail: ghdfurtado@hotmail.com \\ ${ }^{5}$ Mestre; Médico Veterinário; Professor/UNINASSAU/PB. E-mail: leovetleleco@gmail.com \\ ${ }^{6}$ Cadre Medico Educatif, Educatrice Spécialisée, Monitrice Educatrice, Aide Medico Psychologique/Institut \\ Régional du Travail Social, Poitiers, France (IRTS). E-mail: ouriques@hotmail.fr
}

Résumé. La gale notoedric, également appelée "gale du chat", est une maladie parasitaire causée par un acarien appelé Notoedres cati. Il affecte généralement les chats, mais peut également affecter les chiens et les humains. L'objectif de cette revue était de présenter les informations disponibles dans la littérature scientifique sur la gale notoedric, dans le but de contribuer à l'efficacité de l'information et à une meilleure préparation des universitaires vétérinaires. Pour cela, des études et des recherches bibliographiques ont été effectuées, ainsi que des consultations de publications scientifiques dans des bases de données de médecine vétérinaire. Nous pouvons en conclure que la gale notoedric est très contagieuse, généralement par contact direct, et que le meilleur moyen de contrôler cette parasitose consiste à hygiéniser le lieu de vie des chats et à les empêcher de toucher les animaux infectés. Mots-clés: Chats; Acariens; Gale notoedric.

\section{SARNA NOTOÉDRICA: UMA BREVE REVISÃO}

Resumo. A sarna notoédrica, também conhecida como "escabiose do gato", é uma doença parasitária causada por um ácaro denominado Notoedres cati. Comumente afeta os gatos, mas também pode afetar cães e seres humanos. O objetivo desta revisão foi apresentar as informações disponíveis na literatura científica sobre ao sarna notoédrica, visando contribuir com a eficiência de informação e melhor preparo de acadêmicos de veterinária. Para tanto, foram realizados estudo e pesquisas bibliográficas, bem como consultas de publicações científicas em bases de dados da área de medicina veterinária. Podemos concluir que a sarna notoédrica é altamente contagiosa, geralmente por meio do contato direto e a melhor maneira de se controlar esta parasitose é por meio da higienização do local onde os gatos vivem e também evitando que eles tenham contato com animais infectado. 
Palavras-chave: Gatos; Ácaros; Sarna notoédrica.

\section{NOTOEDRIC SCABIES: A BRIEF REVIEW}

Abstract. Notoedric scabies, also known as "cat scabies", is a zoonosis caused by a mite called Notoedres cati. It commonly affects cats, but can also affect dogs and humans. The objective of this review was to present the information available in the scientific literature on the scabies of notoedric, aiming to contribute to the information efficiency and better preparation of veterinary academics. For this, studies and bibliographical research were carried out, as well as consultations of scientific publications in databases of veterinary medicine. We can conclude that notoedric scab is highly contagious, usually through direct contact and the best way to control this parasitosis is through the hygiene of the place where the cats live and also preventing them from having contact with infected animals.

Keywords: Cats; Mites; Notoedric scabies.

\section{SARNA NOTOEDRICA: UNA BREVE REVISIÓN}

Resumen. La sarna notoédrica, también conocida como "escabiosis del gato", es una zoonosis causada por un ácaro denominado Notoedres cati. Comúnmente afecta a los gatos, pero también puede afectar a perros y seres humanos. El objetivo de esta revisión fue presentar la información disponible en la literatura científica sobre el sarna notoédrica, con el objetivo de contribuir con la eficiencia de información y mejor preparación de los académicos de veterinaria. Para ello, se realizaron estudios e investigaciones bibliográficas, así como consultas de publicaciones científicas en bases de datos del área de medicina veterinaria. Podemos concluir que la sarna notoédrica es altamente contagiosa, generalmente por medio del contacto directo y la mejor manera de controlar esta parasitosis es por medio de la higienización del lugar donde los gatos viven y también evitando que ellos tengan contacto con animales infectados.

Palabras clave: Gatos; Ácaros; Sarna notoédrica.

\section{INTRODUCTION}

Dermatose parasitaire, la gale notoedric se développe en raison des acariens qui vivent dans la peau et / ou sous la peau de l'animal hôte. En raison des variations de la durée de l'infestation, de l'intensité de la réaction d'hypersensibilité et de la capacité biologique de l'hôte, la variabilité des manifestations cliniques de la gale notoedric semble limiter la multiplication du parasite (TAYLOR, 2017).

L'exposition des hôtes à ces acariens ainsi que la présence de dermatoses parasitaires chez eux sont fortement associées à des facteurs environnementaux, notamment au contact d'autres animaux et à la présence de zones d'endémie. Bien que les acariens manifestent une préférence pour certains hôtes, ils ne présentent pas une spécificité unique pour eux. Ils peuvent 
également être fixés chez d'autres animaux et présentent un grand potentiel zoonotique pour provoquer des dermatoses chez l'homme (AILEXANDRINO, 2008; OCAÑA, 2003).

Notoedres cati est un acarien parasite trouvé comme chez le chat et le lapin, où des lésions sont observées sur le visage et dans le pavillon des oreilles, ainsi que dans les régions où les poils sont plus fins et plus maigres. Cependant, ceux-ci peuvent se propager à d'autres parties du corps. Avec un degré élevé de démangeaisons, les lésions sont croustillantes et, en raison de l'utilisation des ongles lors du grattage, des lésions traumatiques peuvent apparaître (MONTEIRO, 2017; TAYLOR, 2017).

\section{CARACTÉRISTIQUES MORPHOLOGIQUES ET CYCLE}

Cette morphologie ressemble beaucoup à la gale canine (Sarcoptes scabiei) (SLOSS et al., 1999). Cet acarien a le corps globuleux chez les deux sexes et les mâles et les femelles sont rougeâtres. Le tégument est strié comme dans les Sarcoptes, le visage dorsal à écailles molles et de longues épines minces. Les rejets ambulacraux des jambes 1, 2 et 4 chez les hommes et les femmes des jambes 1 et 2 (FORTES, 1997).

Au cours de son cycle évolutif, la femelle pondeuse met environ 60 œufs dans des galeries qu'elle fouille pendant sa vie, qui dure de trois à quatre semaines, après lesquelles elle meurt (URQHART et al., 1998).

\section{SIGNES CLINIQUES ET TRAITEMENT DES ANIMAUX}

Les infections se manifestent par des lésions squameuses et sèches, avec des croûtes sur les bords des oreilles et du visage, ainsi que sur une peau épaissie et un peu coriace. Le prurit provoqué par cette pathologie est souvent intense et peut entraîner de graves irritations de la région de la tête et du cou en raison des éraflures intenses provoqué par les grattages. En général, les lésions apparaissent généralement en priorité sur les bords médians du pavillon auriculaire, en continuant de se propager aux oreilles, au museau, aux paupières et au cou. Il peut se diffuser par la queue par contact lorsque le chat se nettoie et dort (SMITH, B.P, 1993; URQHART et al., 1998).

En raison de la résistance de cet acarien, il peut vivre quelques jours à l'extérieur de l'hôte et, de cette manière, des objets communs de l'animal tels que brosse à lit, peigne et tondeuse de toilettage (des ustensiles en règle générale) peuvent être de fortes sources de contagion (NOGARI et al., 2004).

Dans la pathogenèse, on observe la destruction du tissu cutané et la formation de papules (FORTES, 1997). Le diagnostic est établi en fonction de l'hôte impliqué, du prurit intense, de 
la localisation de la lésion et de sa dissémination rapide. La reconnaissance des acariens par raclage de la peau, où un seul nid dans une éraflure peut révéler de nombreux acariens (URQHART et al., 1998), en témoigne.

La contamination se produit par contact direct avec un autre animal infecté ou avec des objets contaminés tels qu'une tondeuse à toilettage, une brosse, une serviette, une cage, entre autres (LIMA et al., 2009).

L'acarien "creuse" des tunnels dans les régions les plus profondes de la peau, générant des démangeaisons intenses, symptôme le plus évident de la gale. En outre, il existe également une perte de poils, une anorexie due à une irritation causée par le parasite, une desquamation et une apparence de croûtes, en particulier sur la tête, les oreilles et les pieds, pouvant se propager dans tout le corps de l'animal (MONTEIRO, 2017).

\section{SIGNES CLINIQUES À HUMAOS}

La variété Notoedres cati, à titre exceptionnel, peut affecter l'homme (LEITÃO, 2007). Cependant, les agents étiologiques responsables de la gale chez les animaux domestiques ne pénètrent pas dans la peau humaine et ne se multiplient pas avec le contact possible. Ils ne peuvent déclencher qu'une dermatite (éruption cutanée) temporaire à disparition rapide (RODRIGUES, 2014).

Les signes cliniques présentés chez l'homme sont des taches rougeâtres sur les bras et la poitrine (zones qui entrent normalement en contact avec les animaux) et des démangeaisons intenses (LIMA et al, 2009).

\section{CONCLUSIONS}

La gale notoedric est une dermatose de chat causant des démangeaisons intenses, causée par l'acarien sarcoptiforme. Les acariens peuvent également infester les chiens et peuvent causer des blessures transitoires chez les humains en contact avec des animaux infestés. Nous pouvons donc en conclure que le meilleur moyen de contrôler cette parasitose consiste à assainir l'endroit où vivent les chats et à les empêcher d'entrer en contact avec des animaux infectés.

\section{RÉFÉRENCES}

AILEXANDRINO, M. Sarnas que acometem cães e gatos - Sarna Notoédrica. São Paulo, 2008.

FORTES, E., Parasitologia Veterinária. $3^{\text {a }}$ ed. Cone. São Paulo. 1997. 
LEITÃO, J. P. A sarna Notoédrica. Centro Médico Veterinário da Cabra Figa. 2007.

LIMA, G. S.; ALVES, R. M.; NEVES, M. F. Sarna notoédrica: Notoedres cati. Revista Científica Eletrônica de Medicina Veterinária. Ano VII. n. 12. 2009.

MONTEIRO, S. G. Parasitologia na medicina veterinária. 2. ed. Rio de Janeiro: Roca, 2017.

NOGARI, F.; SOTO, F. R. M.; RISSETO, M. R.; SOUSA, O. Programa de tratamento e controle de doenças parasitárias em cães e gatos de proprietários de baixa renda no município de Ibiúna. Revista ciência em extensão. São Paulo. 2004

OCAÑA, C. F. Dermatosis felinas en colectividades. Pequeños Anim. Dpto. Patología Animal I. HCV Facultad de Veterinaria. Madrid. 2003.

RODRIGUES, T. O. S. Sarna Humana. Dissertação (Mestrado em Ciências Farmacêuticas) UNIVERSIDADE FERNANDO PESSOA. Porto. Portugal. p. 45. 2014.

SLOSS, M.W.; ZAJAC, A.M.; KEMP, R.L. Parasitologia clínica veterinária. $6^{\text {a }}$ ed. Manole. São Paulo. 1999.

SMITH, B.P. Tratado de medicina interna de grandes animais. $1^{\text {a }}$ ed. Manole, São Paulo, 1993.

TAYLOR, M. A. Parasitologia veterinária. 4. ed. Rio de Janeiro. Guanabara Koogan, 2017.

URQHART, G.M.; AMOUR, J.; DUNCAN, J.L.; JENNINGS, F.W. Parasitologia Veterinária. $2^{a}$ ed. Guanabara Koogan. Rio de Janeiro, 1998. 